\title{
The New Circulation Type Sample Pool Design of Online Testing for Impurity Oil Fluorescence in Water
}

\author{
Zhikun Chen \\ College of Electrical Engineering HeBei United University \\ Tang Shan, China \\ Yan Zhou \\ College of Electrical Engineering HeBei United University \\ Tang Shan, China
}

\begin{abstract}
This article has designed a new sample pool for online measures the optical properties of oil compounds which contains in water impurity. The sample pool adopt unique circulation type of sampling technology to makes the continuous on-line detection, the sample pool design including light source, optical system which transmit the detected fluorescent to small fiber spectrometer directly at the same time. To realized the online measurement of impurity oil in water. The experimental results show that the sample pool design is completely feasible.
\end{abstract}

Keywords-component; sample pool; online measurement; the water oil organic impurity

\section{INTRODUCTION}

With the development of material science, and ecological protection, environmental monitoring and so on, all kinds of spectrum detection requirements are increasing, the traditional sample pool need the conditions of stable experiment platform and relative stable temperature to complete the liquid measurement in sample pool. The sample pool has small differences in the aspects of optical path position, angle and cell material, all may affect the accuracy of spectral measurement. Open-top sample pool is not suitable to measure volatile organic solvent sample. Traditional spectrometer can't satisfy the online testing impurity in water oil organic matter. In addition, the cleaning of sample liquid and update are all need manual operation. For unstable experiment area of a large number of sample testing and field operation, the traditional sample pool cannot satisfy the test requirement [1].

With the development of industry, the environmental protection work of surface water has under the great pressure. Oil pollution has become an increasingly important issue. The rapid development of industrial, agricultural production and human life garbage increase rapidly. A lot of pollutants pour into surface water and it harmful to people's health seriously and restricts the sustainable development of national economy. In view of the consumption of residents life, industrial and agricultural production improve continuously and the social

\author{
Yi Zhang \\ College of Electrical Engineering HeBei United University \\ Tang Shan, China \\ foreverzhang8512@163.com \\ Yufeng Zhou \\ College of Electrical Engineering HeBei United University \\ Tang Shan, China
}

increasing attention on water environment protection work, water quality monitoring technology also should be strengthen and improve. Due to the source of oil impurity in water is different; the component difference is very big. Mainly because the different boiling point of fat hydrocarbon, aromatic hydrocarbon and non hydrocarbon make the complex mixture. So people use a variety of methods for its analysis and determination. Fluorescence analysis has the properties of high sensitivity, high selectivity, shortcut, good reproducibility, sampling easily and few sample requirements and has the irreplaceable advantages on the microscope oil measurement [2].

Based on the urgent needs of spectral measurement on all kinds of environmental monitoring in our country, in allusion to the spectrum instrument liquid sample pool existing the shortages; this article designed a new sample pool for online measurement of the water oil impurity organic optical properties. The sample pool adopt unique circulation type of sampling technology to makes the continuous on-line detection, the sample pool design including light source, optical system which transmit the detected fluorescent to small fiber spectrometer directly at the same time. To realized the online measurement of impurity oil in water [3].

\section{THE NEW SAMPLE POOL DESIGN OF ONLINE TESTING FOR IMPUIRY OIL FLUORESCENCE IN WATER}

The diagram of the sample pool design structure has been show in figure 1. The sample cell mainly includes four parts. Respectively are excitation light source, sample cell, optical system and optical fiber probe. 


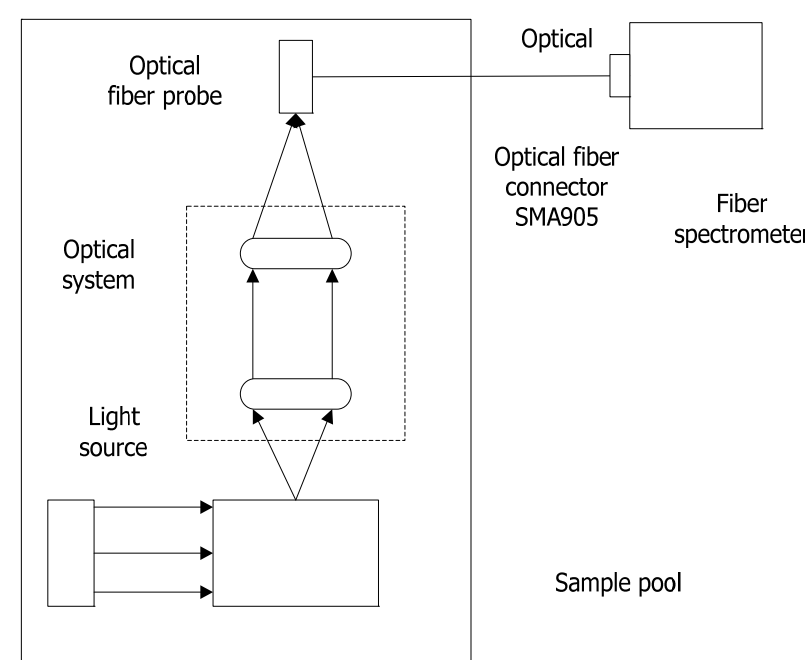

Figure 1. The structure diagram of sample pool design

The working process of the sample cell: Excitation light source illuminate sample pool with parallel light, produced fluorescence was focused by optical system and collected by the optical fiber, and then it transmitted to small fiber spectrometer. The sample cell influx and outfall can realize continuous measurement of liquid.

\section{A. Excitation light source}

According to comparison of the a variety of light source, super high pressure xenon lamp, high pressure mercury lamp, hollow cathode lamp, hydrogen arc lamp in energy spectrum distribution characteristic curve, then choose the high strength, low power consumption, low repetition frequency, narrow pulse width of the high voltage pulse xenon lamp as excitation light source. Pulse xenon lamp and other continuous luminescence light source is different, it can light strongly in a very short time, so often called it flash. Pulse xenon lamp can provide high strength continuous spectrum from ultraviolet to infrared band, produce strong instantaneous power which continuous light source can not, thus obtain strong instantaneous light output, and by adjusting the charge and discharge capacity to change current flash light intensity. Because the way of intermittent flashing, pulsed Xenon has low energy consumption, small temperature rise, low average power, the intensity difference of each flash light is small, low environmental impact, good stability.

This article select the 1100 series FX-1160 short-arc Xenon lamp which produced by EG\&G company production as light source [4-5]. Short-arc xenon is only $1.5 \mathrm{~mm}$, easy to fiber coupling. Short-arc Xenon adopts intermittent flashing (periodicity instantaneous luminescence), low energy consumption, low average power, small temperature rise, little affected by the ambient temperature, besides each flash of light intensity difference is very small and keeping good stability. At the same time it has the characteristics of small heat accumulation, small size, simple operation, and less impact on the environment and surface target is small. Optical radiation wavelength is $150 \mathrm{~nm} \sim 1100 \mathrm{~nm}$. Detection range is $190 \mathrm{~nm} \sim 870 \mathrm{~nm}$. Window material is boron silicate. Maximum flash peak energy is $0.5 \mathrm{~J}$. Average power is $20 \mathrm{~W}$. Operating voltage DC is $350 \sim 1000 \mathrm{~V}$. Life expectancy is greater than $10^{-9}$. FX-1160 structure is shown as figure 2 .

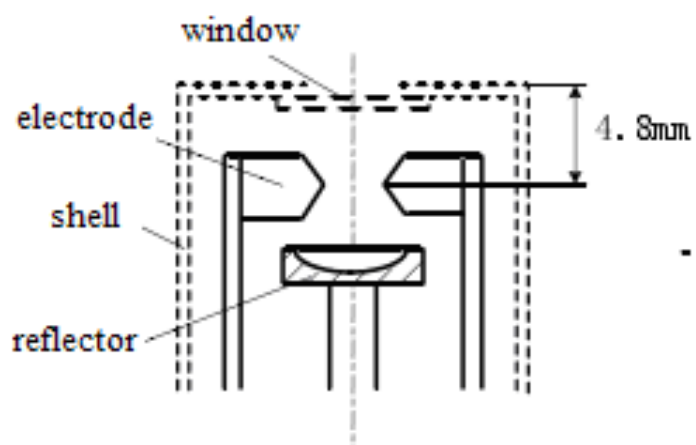

Figure 2. Longitudinal profile

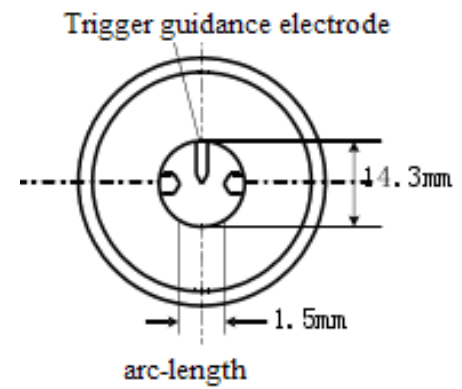

Figure 3. Transverse profile

The structure size of excitation light source in the whole sample pool is shown as figure 4 .

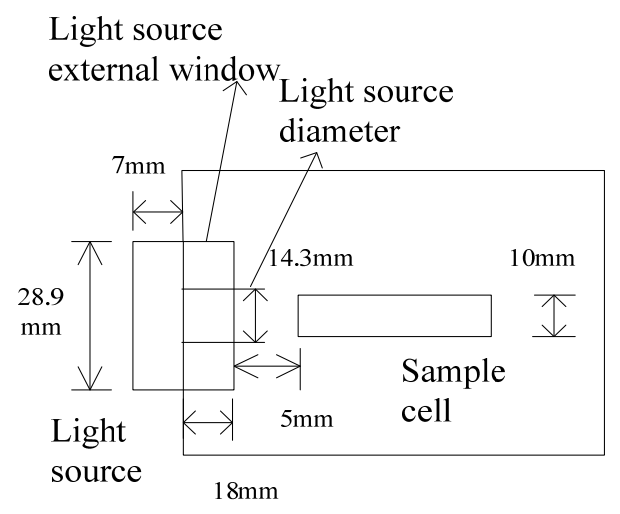

Figure 4. The structure size of excitation light source in the whole sample pool

\section{B. Sample pool structure}

Because of the spectral emission fluorescence of quartz glass materials is weak in the research questions, it is using quartz cell as sample pool. The measure direction of fluorescence is perpendicular to the excited light in order to eliminate the influence on fluorescence of transmission light and stray light. The better suitable shape of sample pool is square or rectangular, stray light interference is much smaller. In order to make quantitative calculations, a fixed volume of the sample pool is $4 \mathrm{ml}$ and wide-height-length is $10 \mathrm{~mm} \times 10 \mathrm{~mm} \times 40 \mathrm{~mm}$. Excitation light intensity is $I_{0}$. 
Transmitted light intensity is $I_{t}$. Fluorescence intensity is $I_{f}$. Sample liquid flows from up to down in inflow and outflow. It is shown as figure 5,6 .

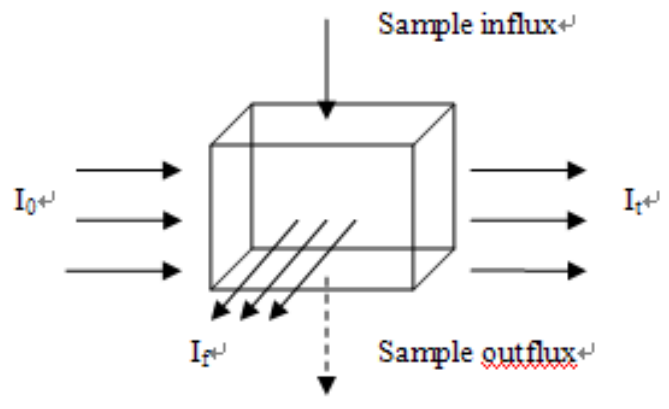

Figure 5. Sample pool structure

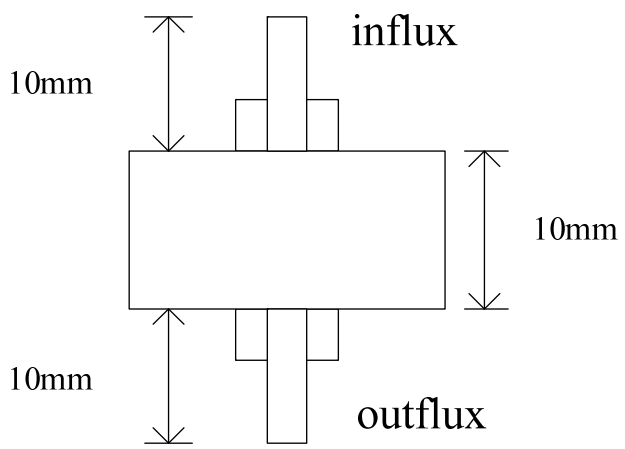

Figure 6. The structure size of sample pool

\section{Optical system}

Optical system is mainly focusing the divergence of fluorescent, facilitating fluorescence collection detection. Because of fluorescence is rendered hemispherical divergence, therefore, optical systems is collimating fluorescence and focusing in importing small fiber spectrometer. Collimating lens, Focusing lens: its material is zinc selenide. They are convex lens. Diameter of $d$ is $38.1 \mathrm{~mm}$. Focal length $\mathrm{f}$ is $71 \mathrm{~mm}$. Optical system structure is shown as figure 7, 8 .

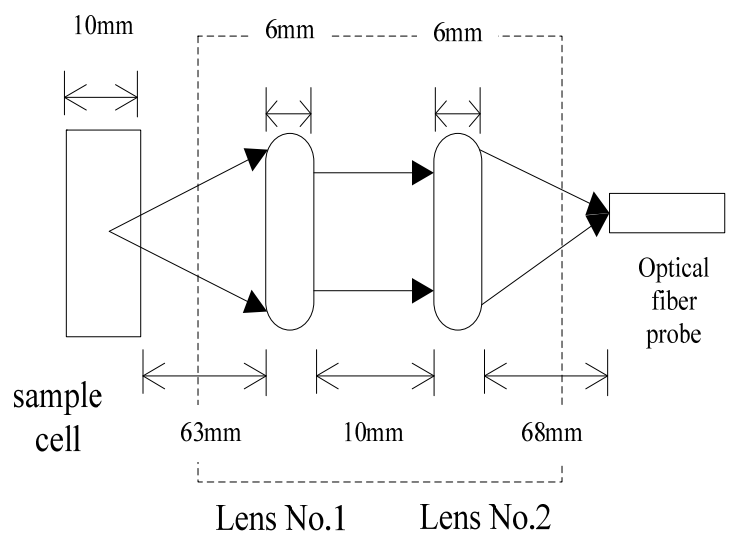

Figure 7. Optical system side Angle diagram

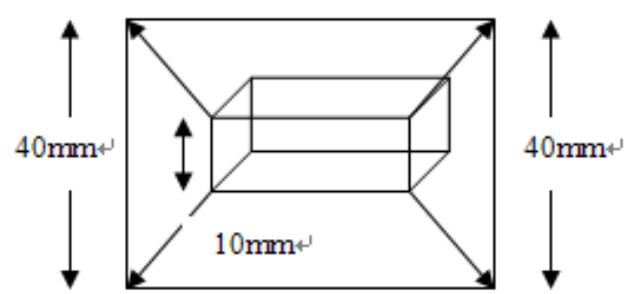

Figure 8. Optical system positive perspective diagram

\section{Optical fiber probe}

Fluorescent collection select optical fiber probe. Consider the good of silica fiber for ultraviolet and visible light transmission characteristics and optical fiber probe sets, this paper adopted the core for $200 \mu \mathrm{m}$, numerical aperture NA is 0.27 , in the near ultraviolet area attenuation of $15 \mathrm{db} / \mathrm{km}$ hard cladding pure quartz optical fiber as fluorescence optical fiber. Fluorescence optical fiber will be seven root fiber bundle collect fluorescence. Probe is made of the fiber core, cladding and coating layer. Fiber core is made of the silicon dioxide. In order to improve the refractive index of the fiber core, it highly doped with a small amount of such as phosphorus pentoxide, germanium dioxide and other materials. Cladding is made of pure silicon dioxide constitute. Polymer materials such as epoxy resin, silicone rubber coated layer as a coating layer of material. Sheathing materials generally use nylon or other organic material [6]. Optical fiber probe structure is shown as figure $9,10,11$.

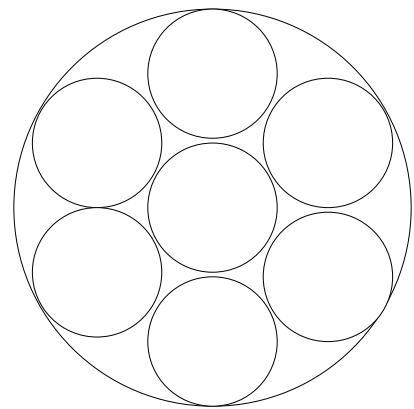

Figure 9. Optical fiber probe transverse section

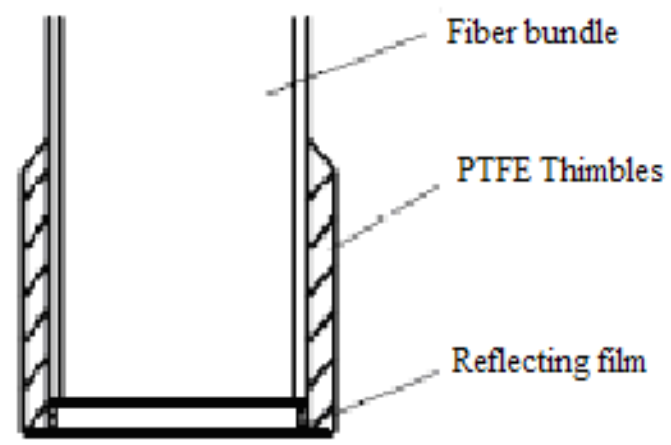

Figure 10. Optical fiber probe longitudinal profile 


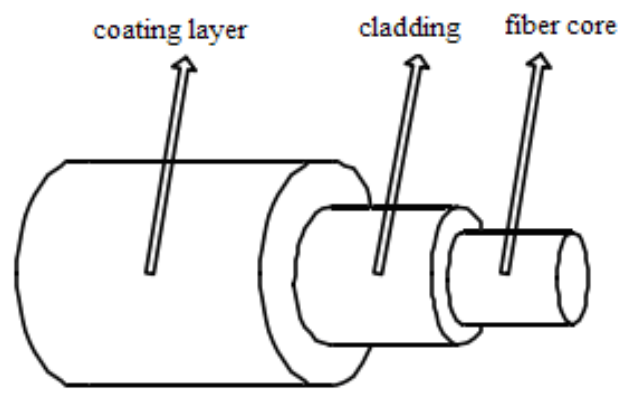

Figure 11. Optical fiber probe structure

III. THE EXPERIMENTAL RESULTS AND ANALYSIS

Take 97 \# gasoline $1 \mathrm{~g}$, and add a small amount of $\mathrm{CCl}_{4}$ and dissolve them in $100 \mathrm{ml}$ volumetric flask. Using $\mathrm{CCl}_{4}$ constant volume [7], then it gets $0.01 \mathrm{~g} / \mathrm{mL}$ standard solution which preserved in room temperature. Take 20 volumetric flasks of 4 $\mathrm{ml}$ (Numbers $1 \sim 20$ ), and add different volume each standard solution. Adding $\mathrm{CCl}_{4}$ to constant volume, then it gets different proportion solution sample. At the 20 samples, $1 \sim 5$ are correction sample, $6 \sim 20$ are forecast sample. Pour the liquid of 20 volumetric flasks into the sample pool in turn to take detection. Three dimensional fluorescence spectra data using PARAFAC decomposition, factoring out the scoring matrix A is the concentration [8-11]. The experimental results as the chart shows:

TABLE I. CONCENTRATION AND ERROR OF PREDICTION SAMPLES

\begin{tabular}{|c|c|c|c|}
\hline Numbers & $\begin{array}{c}\text { Predict } \\
\text { concentration } \\
\mathrm{g} / \mathrm{mL}\end{array}$ & $\begin{array}{c}\text { Detection } \\
\text { concentration } \\
\mathrm{g} / \mathrm{mL}\end{array}$ & $\begin{array}{c}\text { Error } \\
\%\end{array}$ \\
\hline 6 & 0.0003 & 0.000287 & 4.33 \\
\hline 7 & 0.0002 & 0.000191 & 4.5 \\
\hline 8 & 0.0004 & 0.000395 & 1.25 \\
\hline 9 & 0.0012 & 0.001197 & 0.25 \\
\hline 10 & 0.0008 & 0.000798 & 0.25 \\
\hline 11 & 0.0006 & 0.000599 & 0.17 \\
\hline 12 & 0.0003 & 0.000297 & 1 \\
\hline 13 & 0.0002 & 0.000192 & 4 \\
\hline 14 & 0.0007 & 0.000697 & 0.43 \\
\hline 15 & 0.0005 & 0.000495 & 1 \\
\hline 16 & 0.001 & 0.000997 & 0.3 \\
\hline 17 & 0.0009 & 0.000896 & 0.45 \\
\hline 18 & 0.0007 & 0.000694 & 0.9 \\
\hline 19 & 0.0008 & 0.000796 & 0.5 \\
\hline 20 & 0.0006 & 0.000594 & 1 \\
\hline
\end{tabular}

From the results, we can see, the average error of sample pool detection is $1.35 \%$. New sample pool design achieves the target of real-time on-line detection.

\section{CONCLUSION}

New sample cell design will concentrate light source, sample cell, optical system, and optical fiber probe setting at an organic whole. Increasing the circulation type function with small fiber spectrometer to solve the traditional sample pool can't real-time online detection, continuous measurement problem. New sample cell design on water quality of rapid detection and analysis, monitoring of pollutant types, sources, fast recognition pollution nature, providing early warning and tracing accident liability, is important to practical application value.

\section{REFERENCES}

[1] Yang Jing-hai, Wang Li-rong, Gao Ming,et al.Manufacture of the Oil in Water Monitor. Journal of Jilin Normal University. 2008,4:1-3.

[2] Michael J, Mlodzianoski, Joerg. Bewersdorf. Advances in Three Dimensional Super Resolution Fluorescence Localization Microscopy. Biophysical Journal.2011,3:142.

[3] Autrev Tom, Foster Nancy, Hopkins Derek, Price John. Tunable Ultraviolet Visible Photoacoustic Detection: Analysis of the Sensitivity and Selectivity Provided by a Xenon Flash Lamp Analytical Chemical Acta.2001, 434(2):217-222.

[4] Kaifeng Zhou, Shanghai: Regenerative heating furnace control model research, Shaihai Jiaotong University, 2008.

[5] A.Robert, Capobianco.Design Considerations for High-stability Pulsed Light Systems. Perkin-Elmer Optoelectronics.2001:1-6.

[6] Huang Jun,Zhao Wen qi,Zhao Rong, Ding Li yun .Research on Fiber Optic Biosensor with High Precision.Journal Of Wuhan University Of Technology. 2010,21:30-31.

[7] M.H. Chowdhury,K. Ray,J.R. akowicz. The Use of Aluminum Nanostructures in Plasmon-Controlled Fluorescence Applications in the Ultraviolet Toward the Label-Free Detection of Biomolecules. Comprehensive Nano science and Technology.2011,4:215 255.

[8] Rasmus Bro. PARAFAC. Tutorial and application. Ctzemometrics and Intelligent Laboratory System, 1997,35:149-151

[9] KOWALCZUK P,COOPER W J,DURAKO M J,et al Characterization of dissolved organic matter fluorescence in the South Atlantic Bight with use of PARAFAC model: relationships between fluorescence and its components, absorption coefficients and organic carbon concentrations. Marine Chemistry,2010,118 (12): 22-36.

[10] STEDMON C A, BRO R, Characterizing dissolved organic matter fluorescence with parallel factor analysis: atutorial. Limnology and Oceanography: Methods.2008 .6:572-579.

[11] STEDMON C A, MARKAGER S, Resolving the variability in dissolved organic matter fluorescencein atemperate estuary andits catchment using PARAFAC analysis. Limnology and Oceanography, 2005, 50 (2) :686-697. 